\title{
A Sample Holder with integrated laser optics for an ELMITEC Photoemission Electron Microscope
}

\author{
L. Gierster,${ }^{1}$ L. Pape,${ }^{1} \quad$ A. A. Ünal, ${ }^{1}$ and F. Kronast ${ }^{1,{ }^{*}}$ \\ ${ }^{1}$ Helmholtz-Zentrum Berlin für Materialien und Energie, Albert-Einstein Str. 15, 12489 Berlin, Germany \\ ${ }^{*}$ Corresponding author
}

\begin{abstract}
We present a new sample holder compatible with ELMITEC Photoemission Electron Microscopes containing an optical lens and a mirror. With the integrated optical elements a laser beam is focused from the back side of the sample at normal incidence, yielding a minimum spot size of about $1 \mu \mathrm{m}$. This opens up new possibilities for local laser excitations in PEEM experiments such as imaging all-optical magnetization switching at a small length scale.
\end{abstract}

\section{Introduction}

Excitations by ultra-short laser pulses are a research topic with increasing interest during the past years, ranging from laser-induced demagnetization and magnetic switching in thin films [1] to the excitation and observation of plasmonic waves at the interface between a metal and a dielectric [2]. Photoemission Electron Microscopy (PEEM) is a powerful technique comprising both, the sensitivity to electrical near fields for the investigation of plasmonic waves [3], and a magnetic sensitivity with high lateral resolution by exploiting the (X-ray) magnetic circular dichroism [4, 5]. Thus an increasing number of PEEM instruments have a laser system attached. One difficulty in implementing the laser beam to experiments is that the extractor lens of the instrument is placed at a small distance to the sample surface, which prevents mounting optical elements directly in front of the sample. A common approach is therefore a grazing incident laser beam. However, with this geometry the spot size is enlarged and for out-of-plane magnetized films the angular momentum transfer of circular polarized laser light to the sample is attenuated. It has been proposed that circular polarized laser light creates an effective magnetic field parallel to the wave vector of laser light due to the inverse Faraday Effect, which may be responsible for the laser helicity dependence of alloptical magnetization switching in ferrimagnetic thin films with out-of-plane magnetization such as GdFeCo [6, 7]. Furthermore the time dependent observation of propagating plasmonic waves by means of two photon photoemission is complicated, because fringe patterns recorded at grazing incidence depend on the propagation directions of plasmonic wave and laser light [8]. While at normal incidence of laser light the observed fringe patterns allow for direct observation of the plasmonic wave packets [9].

A normal incidence laser beam has been achieved by inserting a small mirror inside the back focal plane of the extractor lens [9]. An alternative approach is to bring the light from the back side of the sample. The advantage of this approach is that a focusing lens can be mounted much closer to the sample to achieve a smaller laser spot size. The small spot size can be used for investigating all-optical switching at a small length scale or as a local heat source to investigate the spin Seebeck effect [10] in PEEM, for instance.

In this article, we present a new sample holder containing an optical mirror and a lens, focusing a laser beam at normal incidence from the back side to the sample, yielding a laser focus of 3-5 $\mu \mathrm{m}$. First experiments with the new sample holder have been carried out at the Spinresolved Photoelectron Emission Microscope (SPEEM) [11] at Helmholtz-Zentrum Berlin (HZB) Bessy II synchrotron facility on a thin film of ferrimagnetic TbFeCo. Furthermore a time resolved pump-probe experiment in a $\mathrm{GdFeCo}$ film is presented. Our results illustrate the feasibility to change the magnetic state of the material alloptically at a length scale of few microns.

\section{Design}

Figure 1 shows the schematic of the sample holder. The ELMITEC compatible base plate contains a mount for the small mirror, which is a protected silver coated $5 \mathrm{~mm}$ square Edmund Optics mirror. The sample is placed on an iron yoke with a gap just below the sample. Applying a current to the coils that are installed to the iron yoke is possible via the contact springs at the bottom of the base plate. This allows in-plane magnetic field of some tens of $\mathrm{mT}$ to be generated during measurements, as already described in Ref. [12]. In the gap below the sample a lens holder can be placed, holding an Edmund Optics plano-convex lens (NIR I coated) with a diameter of $3 \mathrm{~mm}$ and an effective focal length of $3 \mathrm{~mm}$ (for $\lambda=590 \mathrm{~nm}$ ). The laser beam is focused from the back side of the sample and passes through the transparent substrate before hitting the investigated film.

In our experiments we use double-side polished 0.5 mm-thick $\mathrm{MgO}$ substrates and the Femtolasers Scientific XL Ti:sapphire oscillator with a central wavelength of $\lambda=800 \mathrm{~nm}$. Considering this wavelength and the refractive index of our $\mathrm{MgO}$ substrate we calculated that the spot size reaches a minimum value of $0.36 \mu \mathrm{m}$ at a distance of 2.7 
$\mathrm{mm}$ from the lens. However, this spot size is diffraction limited by the small aperture given by the lens diameter of $3 \mathrm{~mm}$. Accordingly a minimal spot size of about the size of $\lambda$ can be expected, corresponding to the full width of half maximum of the Airy disc. As already mentioned, an inplane magnetic field can be optionally combined with the laser excitation. This may be a useful option in investigating laser induced magnetization switching in materials with inplane magnetic anisotropy. In principle, also an out-of-plane magnetic field would be achievable by placing a solenoid underneath the sample, leaving place for the light to hit the mirror.

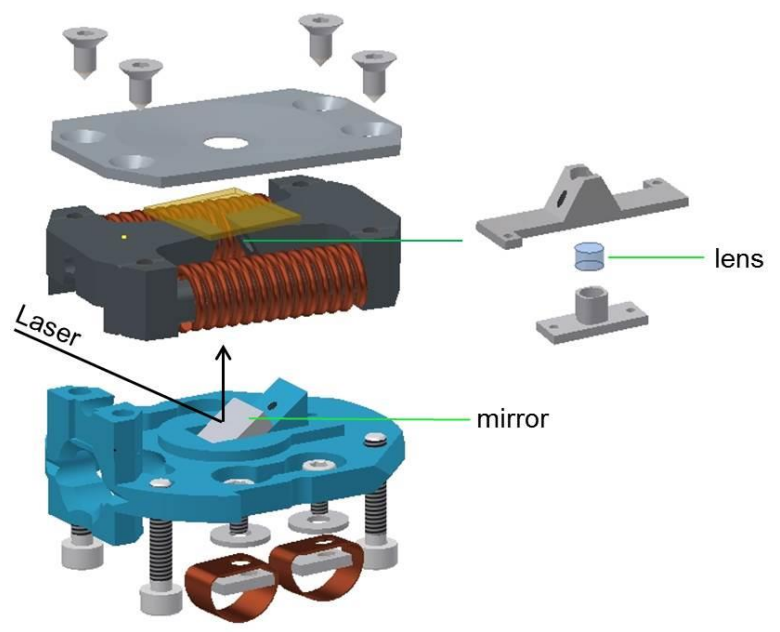

FIG. 1. (Color online). Schematic of the sample holder where a mirror and a lens (focal length $3 \mathrm{~mm}$ ) are implemented to focus the incoming laser light to the back side of the sample.

\section{Experimental Results}

\section{A. Laser spot characterization and alignment}

Figure 2 a) shows that even for low intensities the laser spot can be observed via the photoelectron yield in PEEM, which makes it possible to align the laser beam for experiments. We checked this for different films, such as TbFeCo (capped with Pt), GdFeCo (capped with $\mathrm{Al}$ ) and $\mathrm{Fe}$ (capped with $\mathrm{Al}$ ). The investigated film must be thin, because laser light will be extenuated when it enters from the back side of the sample. For example, for an about 20 $\mathrm{nm}$-thick film of TbFe the extinction is as high as $34 \%$ for $\lambda=800 \mathrm{~nm}$ [13]. Thus the investigated film may not be thicker than some ten $\mathrm{nm}$ in order to have a well-defined light induced effect. Figure 2 b) shows that the intensity distribution of the emitted electrons has a Gaussian shape. This is not necessarily expected because the energy of laser photons $(1.55 \mathrm{eV}$ at $\lambda=800 \mathrm{~nm})$ is much smaller than the typical work function of metals. Multi-photon excitation and surface plasmons have to be considered as mechanism of electron emission. We observed emitted electrons with kinetic energies of up to $20 \mathrm{eV}$. This is a clear indicator for the presence of electrical near field enhancement due to the excitation of surface plasmons [14]. Therefore the imaged electron intensity distribution might differ to some extend from the intensity profile of the laser spot. From Figure $2 \mathrm{~b}$ ) we determine the apparent spot size of the elliptical spot to about $3 \mu \mathrm{m}$ (short axis) and $5 \mu \mathrm{m}$ (long axis), respectively.

Although the position of the sample is fixed with respect to the lens once it is mounted, we found that by changing the laser incidence angle slightly we could laterally move the laser spot. A manageable way to do this is to alter the tilt correction of the instrument. By this we could laterally position the laser on the sample, whereas the spot size did not change noticeably.
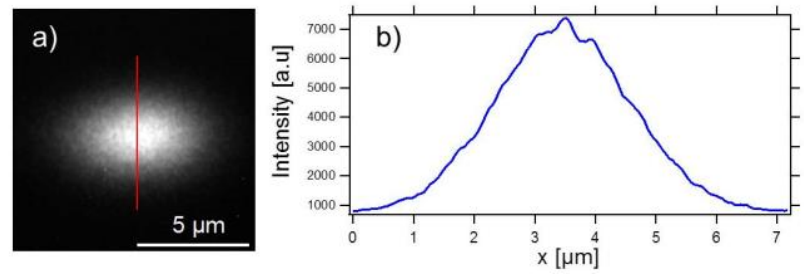

FIG. 2. (Color online). a) Direct photoemission from the laser spot measured by PEEM. b) Intensity distribution along the line shown in a), yielding a Gaussian profile with a full width of half maximum of $3 \mu \mathrm{m}$.

\section{B. First application: Laser induced magnetization switching in $\mathrm{TbFeCo}$}

As an alternative characterization of the laser spot we performed first experiments on a $20 \mathrm{~nm}$-thick $\mathrm{Tb}_{22} \mathrm{Fe}_{69} \mathrm{Co}_{9}$ film. The ferrimagnetic film with out-of-plane anisotropy was deposited by magnetron sputtering onto an $\mathrm{MgO}$ substrate and capped by a $1.8 \mathrm{~nm}$-thick Pt layer in order to prevent oxidation. Figure 3 shows the magnetic state of $\mathrm{Tb}$ by exploiting the XMCD contrast at the $\mathrm{Tb}_{5} \mathrm{M}_{5}$-absorption edge. The magnetic film was previously saturated, corresponding to a red magnetic contrast. In panel a) we recorded a magnetic image during laser excitation, i.e. the image was acquired with laser applied at $8 \mathrm{~mW}$ laser power, $130 \mathrm{fs}$ pulse duration, and $1.25 \mathrm{MHz}$ repetition rate, but without synchronization between laser and y-ray pulses. Therefore it represents an average over the temporal magnetization dynamic which is induced repetitively with each laser pulse.

The magnetic state is changed only in a small region confined by the size of the laser spot. In the central area of panel a) the magnetic contrast nearly vanishes, indicating a laser induced temporal demagnetization or magnetic fluctuations, whereas in the area surrounding the center a ring-shaped magnetic structure forms. In the outer ring the magnetization is switched with respect to the initial state, corresponding to a blue magnetic contrast, whereas the inner ring has again red magnetic contrast. If the laser is switched off, the magnetization in the center relaxes to disordered band domains, displayed in panel b). Ramping the laser power to zero allows the magnetization to relax into a more ordered state, as shown in panel c). We suggest that the formation of concentric rings with alternating outof-plane magnetization is due to dipolar coupling. The outer blue ring emerges due to the dipolar field of the saturated 
film and the inner red ring starts when the dipolar field of the blue ring exceeds that of the saturated film and so on. For $\mathrm{TbFeCo}$ it was shown before that dipolar coupling has a strong influence on the formation of the magnetic state after laser irradiation at this length scale [15, 16].

From Figure 3 it can be seen that the shape and size of the region, which is affected by the laser corresponds to the electron yield displayed in Figure 2. This indicates that the laser footprint matches quite well with photoelectron distribution measured by PEEM.
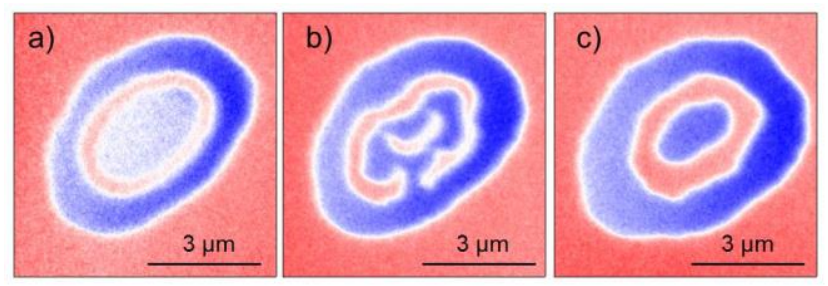

FIG. 3. (Color online). Magnetic state of TbFeCo according to the $\mathrm{XMCD}$ contrast at the $\mathrm{Tb}_{\mathrm{M}}$-absorption edge: a) Static measurement during laser excitation with repetitive 130 fs laser pulses (repetition rate $1.25 \mathrm{Mhz}$ ). b) and c): After laser irradiation (details please see text). Red and blue correspond to the two antiparallel out-of-plane magnetization directions, white lines indicate the presence of a domain wall.

\section{Time resolved pump-probe experiment in GdFeCo}

By synchronizing laser and X-ray pulses and delaying the laser pulse with respect to the X-ray pulse, time-resolved pump-probe experiments can be carried out at SPEEM with a temporal resolution of 30-50 ps. The new sample holder can be used to investigate the laser induced magnetization dynamics on the length scale of few micrometers. We performed a time resolved measurement in a $\mathrm{Gd}_{22} \mathrm{Fe}_{69} \mathrm{Co}_{9}$ sample, grown in the SPEEM preparation chamber by thermal evaporation. Figure 4 presents the magnetic contrast at the $\mathrm{Gd} \mathrm{M}_{5}$-absorption edge, recorded at different delays between laser and x-ray pulse are presented. Starting with a magnetically saturated state (blue color), the magnetization of the Gd sublattice is quenched after the excitation with the femtosecond laser pulse (time 0), indicated by vanishing (white) magnetic contrast. At a delay of $1 \mathrm{~ns}$, the magnetic contrast inside the irradiated area is already slightly red, indicating that the magnetic state is locally reversed by the laser and evolves into a reversed domain, similar to the observations in Ref [10]. At $800 \mathrm{~ns}$ the reversed domain has already shrunk considerably and it collapses and disappears at delays longer than $5 \mathrm{~ms}$. This is shown by the last image in Fig. 4 .

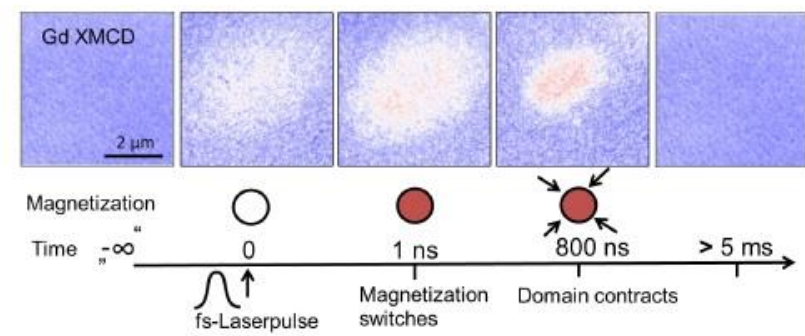

FIG. 4. (Color online) Time resolved pump-probe experiment in GdFeCo. The magnetic state of the Gd sublattice is measured by exploiting the XMCD contrast at the $\mathrm{Gd} \mathrm{M}_{5}$-absorption edge. Before laser irradiation (time "- $\infty$ "), the sample is in a magnetically saturated state encoded by blue color. After the excitation with a 130 fs-laser pulse the magnetization is locally demagnetized, indicated by vanishing magnetic contrast (time 0 ). The magnetic state evolves into a reversed domain which collapses at a time scale of milliseconds.

We attribute the instability of the reversed domain to the lack of pinning sites in the in-situ grown $\mathrm{Gd}_{22} \mathrm{Fe}_{69} \mathrm{Co}_{9}$ film.

\section{Summary}

We presented a new sample holder for PEEM focusing laser light from the back side to the sample by an integrated mirror and a focusing lens. We could demonstrate a laser spot size of 3-5 $\mu \mathrm{m}$ on the sample, which is close to the expected spot size of about $1 \mu \mathrm{m}$. Furthermore we showed experimental data which prove that it is possible to change the magnetic state of a ferrimagnetic thin film of $\mathrm{TbFeCo}$ at a length scale corresponding to this laser spot size. In a GdFeCo thin film a pump-probe experiment at a length scale of few micrometer could be performed. The results suggest that PEEM together with the presented sample holder can give new insights into the field of all-optical switching at a small length scale. Owing to the normal incidence geometry, the helicity dependence of the laserinduced switching can also be investigated.

\section{Acknowledgments}

We thank Dr. Florin Radu for the preparation of the TbFeCo sample and Kerstin Kalus for her help with the technical drawings.

${ }^{1}$ A. Kirilyuk, A. V. Kimel and Th. Rasing, Review of Modern Physics 82, 2731 (2010).

${ }^{2}$ M. S. Tame, K. R. McEnery, S. K. Özdemir, J. Lee, S. A. Maier and M. S. Kim, Nature 9, 329-340 (2013).

${ }^{3}$ M. Cinchetti, A. Gloskovskii, S. A. Nepjiko and G. Schönhense, Physical Review Letters 95, 047601 (2005).

${ }^{4}$ C. M. Schneider and G. Schönhense, Reports on Progress in Physics 65, R1785-R1839 (2002).

${ }^{5}$ T. Nakagawa and T. Yokoyama, Review of Scientific Instruments $\mathbf{7 8}$, 023907 (2007).

${ }^{6}$ C. D. Stanciu, F. Hansteen, A. V. Kimel, A. Kirilyuk, A. Tsukamoto, A. Itoh and Th. Rasing, Phyiscal Review Letters 99, 047601 (2007).

${ }^{7}$ K. Vahaplar, A. M. Kalashnikova, A. V. Kimel, D. Hinzke, U. Nowak, R. Chantrell, A. Tsukamoto, A. Itoh, A. Kirilyuk and Th. Rasing, Physical Review Letters 103, 117201 (2009).

${ }^{8}$ N.M. Buckanie, P. Kirschbaum, S. Sindermann and F.-J. Meyer zu Heringdorf, Ultramicroscopy 130, 49-53 (2013). 
${ }^{9}$ P. Kahl, S. Wall, C. Witt, C. Schneider, D. Bayer, A. Fischer, P.

Melchior, M. Horn-von Hoegen, M. Aeschlimann and F.-J. Meyer zu Heringsdorf, Plasmonics 9, 1401-1407 (2014).

${ }^{10}$ K. Uchida, S. Takahashi, K. Harii, J. Ieda, W. Koshibae, K. Ando, S. Maekawa and E. Saitoh, Nature 455, 778-781 (2008).

${ }^{11}$ F. Kronast, J. Schlichting, F. Radu, S.K. Mishra, T. Noll, H.A. Dürr, Surface and Interface Analysis 42, 1532-1536 (2010).

${ }^{12}$ O. Sandig, J. Herrero-Albillos, F. M. Römer, N. Friedenberger, J.

Kurde, T. Noll, M. Farle and F. Kronast, Journal of Electron

Spectroscopy and Related Phenomena 185, 365-370 (2010).

${ }^{13}$ A. Hassdenteufel, B. Hebler, C. Schubert, A. Liebig, M. Teich,

M. Helm, M. Aeschlimann, M.Albrecht and R. Bratschitsch, Advanced Materials 25, 3122-3128 (2013).

${ }^{14}$ S. E. Irvine, A. Dechant and A. Y. Elezzabi, Physical Review Letters 93, 184801 (2004).

${ }^{15}$ T. Osagawara, N. Iwata, Y. Murakami, H. Okamoto and Y. Tokura, Applied Physical Letters 94, 162507 (2009).

${ }^{16}$ M. Finazzi, M. Savoini, A. R. Khorsand, A. Tsukamoto, A. Itoh, L.

Duo, A. Kirilyuk, Th. Rasing and M. Ezawa, Physical Review Letters

110, 177205 (2013). 